This is the accepted version of an article published by the American Speech-Language Hearing Association (ASHA) in Language, Speech, and Hearing Services in Schools (C) 2021. The manuscript is reprinted here from ASHA and is further available online:

doi.org/10.1044/2021 LSHSS-21-00064

\title{
Functional communication abilities in youth with cerebral palsy: Association with impairment profiles and school-based therapy goals
}

\author{
Clare Koopmans, M.S. ${ }^{1}$, Ashley Sakash, M.S. ${ }^{1}$, Jennifer Soriano, M.S. ${ }^{1,2}$, \\ Helen L. Long, Ph.D. ${ }^{1}$ \& Katherine C. Hustad, Ph.D. ${ }^{1,2}$
}

\footnotetext{
${ }^{1}$ Waisman Center University of Wisconsin - Madison, Madison, WI

${ }^{2}$ Department of Communication Sciences and Disorders, University of Wisconsin-Madison, Madison, WI
}

This study was funded by grant R01 DC009411 from the National Institute on Deafness and Other Communication Disorders, National Institutes of Health. Support was also provided by a core grant to the Waisman Center, U54 HD090256, from the National Institute of Child Health and Human Development, National Institutes of Health.

The authors have no financial relationships relevant to this article to disclose. The authors have no conflicts of interest to disclose.

Short title: Functional communication, impairments, and school intervention in CP

\section{Corresponding Author:}

Katherine Hustad

University of Wisconsin - Madison

1500 Highland Ave.

Madison, WI 53706

kchustad@wisc.edu

(608) 265-9977 


\begin{abstract}
Aim. The aim of this study was to examine the relationship between functional communication skills, underlying speech, language, and cognitive impairments, and school-based speech pathology services in students with cerebral palsy (CP).

Method. Thirty-five participants with CP who had active Individualized Education Programs (IEPS) were classified according to the Communication Function Classification System (CFCS). Participants completed laboratory assessments of speech, receptive language, executive functioning, and non-verbal cognition. Each participant's speech and language IEP goals were coded into treatment units, then categorized into seven, mutually exclusive target goal areas. Non-parametric analyses were employed to examine differences among CFCS groups in the number of deficit areas and the number of goal areas from the IEP. Descriptive analyses were used to evaluate the extent to which deficit areas and goal areas in the IEP co-occurred by CFCS level.

Results. Those in more involved CFCS levels demonstrated more severe speech, receptive language, and cognitive impairments. However, there were no significant differences in the number of deficit areas across CFCS group. Regardless of CFCS level, there were no differences in the number of treatment goals specified in the IEP. Literacy was the only goal area addressed across all CFCS levels. Those in the most involved CFCS levels had AAC goals, but those with more moderate restrictions on functional communication who also had markedly reduced speech intelligibility did not typically have speech or AAC goals.

Interpretation. Individuals with CP across CFCS levels demonstrate variability in their underlying deficit profiles, suggesting that measures of both functional communication and of underlying deficits are necessary to provide a complete picture of communication needs. Literacy goals were common across all CFCS levels, but AAC goals were limited to the most severely involved individuals, suggesting that continuing education may be necessary to support SLPs in developing treatments involving the integration of AAC and speech to foster functional communication at school.
\end{abstract}

Supplemental Material: https://doi.org/10.23641/asha. 16968073 


\section{Functional communication abilities in youth with cerebral palsy: Association with impairment profiles and school-based therapy goals}

Cerebral palsy (CP) is the most common motor disorder in childhood. Children with CP may experience differences in sensation, perception, cognition, communication, and behavior in addition to the hallmark feature of motor impairment. Of those with a CP diagnosis, studies suggest that up to $80 \%$ may have delayed or disordered speech production (Mei et al., 2020) and 55\% may have reduced functional communication skills across environments (Kristoffersson et al., 2020). Communication limitations may lead children with CP to have lower participation levels in leisure activities, a more restricted set of daily activities, fewer friendships, and fewer meaningful communication opportunities at school when compared to their typically developing peers (Majnemer et al., 2008; Mehraban et al., 2016; Raghavendra et al., 2011).

The International Classification of Functioning, Disability and Health for Children and Youth (ICF-CY) framework has shifted the focus of assessment and treatment from a primarily biological or impairment focused approach to include a broader psycho-social perspective that considers the functional impact of the condition on the individual's participation in life (Westby \& Washington, 2017; World Health Organization [WHO], 2007). This shift encourages professionals to consider children from a holistic perspective and recognize their dynamic functional profiles in real life as well as the underlying impairments in body functions and structures that may be associated with different conditions.

A key challenge for characterizing participation and functional abilities is measurement. Because participation occurs in the milieu of real-world environments, it is difficult to measure directly. Thus, indices of participation often involve indirect measures based on the report of others. These measures can involve ratings of particular behaviors using Likert-type scales, or classifications based on observed or reported performance. One such tool that seeks to classify functional communication abilities in individuals with CP is the Communication Function Classification System (CFCS) (Hidecker et al., 2011). The CFCS is a five-level scale designed to classify the effectiveness of everyday communication abilities based on the individual's performance as a sender of information and a receiver of information using any and all modes of communication available (see Table 1). The CFCS does not consider underlying impairments that may be responsible for functional deficits, and therefore has some limitations with regard to its utility in directing treatment. For example, those who are Level III communicators on the CFCS, would be judged as effectively able to communicate as both a sender and receiver of information with familiar partners but not consistently with unfamiliar partners in most environments. The reasons for difficulties with unfamiliar partners could vary greatly (e.g., speech intelligibility deficits, language impairment, intellectual disability) and without information regarding the specific abilities and challenges that underlie the functional profile it is difficult, if not impossible, to develop specific treatment goals to improve functional abilities. Westby \& Washington (2017) suggest that the integration of knowledge about an individual's underlying impairments with a description of that individual's functional participation in the outside world is what creates a complete picture of daily life - and in turn, a complete picture from which a comprehensive treatment plan can be created to maximize individual growth. Thus, there is a need to examine underlying speech and language ability profiles (impairments) that are directly measured using standard clinical tools in the context of the CFCS to further our understanding of how underlying abilities in the speech and language domain translate to functional communication skills. Extant studies suggest that as dysarthria severity 
increases, as indicated by the Viking Speech Scale, CFCS levels tend to increase, reflecting reduced functional communication abilities (Mei et al., 2014). Similarly, children with intellectual disability are more likely to have reduced functional communication abilities on the CFCS (Choi et al., 2018; Himmelmann et al., 2013). However, studies have also indicated that there is variability among cognitive, speech, and language profiles observed within different CFCS levels, supporting the idea that different ability profiles can underlie the different CFCS levels.

\begin{tabular}{ll} 
Table 1. & Communication Function Classification System (CFCS) levels for individuals with CP per Hidecker et al., 2011. \\
\hline Level & Definition \\
\hline I & Effective sender and receiver with unfamiliar and familiar partners. \\
II & Effective but slower paced sender and/or receiver with unfamiliar and/or familiar partners. \\
III & Effective sender and receiver with familiar partners. \\
IV & Inconsistent sender and/or receiver with familiar partners. \\
V & Seldom effective sender and receiver even with familiar partners. \\
\hline
\end{tabular}

When considering the interplay between real-life communication skills and underlying communication impairments in individuals with $\mathrm{CP}$, one environment that plays an essential role across developmental domains is school. In school, students are provided a variety of real-life, functional communication opportunities amongst peers and adults, while simultaneously working toward academic achievement, which almost always involves some type of communication. In a school environment, underlying impairments in domains such as language, cognition, executive function (EF), and speech production are essential to evaluate, as they have direct implications for a child's success, not only with communication, but also with academic performance and eligibility for special education services. Similarly, a child's functional performance is equally important to consider, as authentic participation in one's environment promotes a sense of belonging (Bourke-Taylor et al., 2018). Considering that half or more of children with CP will have some type of communication difference, functional limitation, or speech deficit (Bax et al., 2006; Kristoffersson et al., 2020), it is likely that many will require special education programming with a speech-language pathologist (SLP) to promote functional and academic communication growth across environments. Thus, educational documents such as the Individualized Education Plan (IEP) have some potential to shed light on how those with $\mathrm{CP}$ are being served in the school environment with regard to communication needs.

A small number of studies have examined the types and quality of IEP goals for other schoolaged populations with communication disorders, such as those with speech sound disorders, autism spectrum disorder, and word-reading difficulties (Farquharson et al., 2014); however, only a few studies have focused on the nature of school-based speech-language pathology services for children with CP. Hustad and Miles (2010) examined speech and language IEP goals for children ages 4-5 with $\mathrm{CP}$ by determining if children who could benefit from augmentative and alternative communication (AAC) systems actually had IEP goals that incorporated the use of AAC. Results indicated that of the $95 \%$ of children in the study who could benefit from AAC use, only $57 \%$ had one or more AAC-focused goals written in their IEP, demonstrating that young children with AAC needs may be underserved in school settings (Hustad \& Miles, 2010). Similarly, another study examined the types of communication-based goals that individuals with complex communication needs - further defined as those ages 5-20 years old with autism spectrum disorder, CP, or an intellectual disability-receive in their IEPs (Klang et al., 2016). Results showed that individuals with complex communication needs across etiologies were less likely to have IEP goals focusing on participation, such as using communication skills to interact with peers and adults and to participate in classroom-related and 
leisure activities. As a result, Klang and colleagues asserted that "a shift towards participation goals is not fully realized" in this population (p. 46). Instead, most goals were in the areas of learning and applying knowledge and communication skills without reference to a real-life application of those skills. There is a clear need to understand how the wide-ranging needs specific to individuals with CP in the domain of speech and language are being prioritized in intervention. This information has important implications for ensuring that the communication needs of children with CP are being addressed.

The purpose of the present study was to investigate associations between functional communication as categorized by CFCS level and underlying deficits in speech, cognition, EF, and language, thereby examining the relationship between impairments in body structures and function and activities/participation within the ICF-CY framework. We further sought to determine what types of speech and language goals were prioritized in school for students with different CFCS levels, with an emphasis on identifying similarities and differences in goals based on functional communication abilities (CFCS level) and underlying impairment profiles. Our research questions were as follows:

1) For each level of the CFCS, what are the associated underlying deficits in speech, cognition, EF, and language? Are there differences in patterns of underlying impairments observed in individuals with different functional communication profiles?

2) For each level of the CFCS, what types of speech and language goals and objectives are identified in the IEP? Are there differences among the types of goals and objectives targeted in school for those with different functional communication profiles?

We hypothesized that individuals within each CFCS level would have a range of underlying abilities in speech, cognition, EF, and language, owing to the fact that different constellations of deficits can lead to similar functional communication challenges. Likewise, we expected that a variety of underlying deficits within each CFCS level would lead to a diverse range of speech and language goals targeted in school, but that there would be a close match between goals targeted and underlying deficit areas.

\section{Method}

\section{Participants}

Participants in this study were selected from a larger cohort of individuals in an existing longitudinal study on communication development in $\mathrm{CP}$, which required only that participants have a medical diagnosis of CP. Ethical approval was obtained through the University of Wisconsin-Madison Educational and Social / Behavioral Sciences Institutional Review Board. All individuals in the larger cohort who made at least one in-person visit for the longitudinal study between August 1, 2018 and January 2, 2020 were considered as potential participants in the current study. This timeframe was designed to capture as many participants in the larger cohort as possible while also providing the most recent assessment and IEP data. Participants with other coexisting neurological disorders in addition to $\mathrm{CP}$ were not excluded from participation.

Sixty-three participants had at least one visit within the proposed data collection window. A research SLP contacted primary caregivers of all potential participants to solicit participation and request a copy of the participant's most recent IEP document. Of the 63 families, 13 did not respond, 2 elected not to participate, 11 indicated that their child did not have an IEP at school, and 37 responded 
confirming an interest in participation and an active IEP at school. Of these 37, two were excluded: one participant did not have any speech and language goals, and the other participant's family provided a special education progress report instead of the complete IEP documentation.

Table 2. Demographic characteristics of children with CP by sex, co-diagnoses, and GMFCS

\begin{tabular}{|c|c|c|c|c|c|}
\hline & $\begin{array}{l}\text { CFCS I } \\
n=12\end{array}$ & $\begin{array}{c}\text { CFCS II } \\
n=13\end{array}$ & $\begin{array}{c}\text { CFCS III } \\
n=4\end{array}$ & $\begin{array}{c}\text { CFCS IV } \\
n=3\end{array}$ & $\begin{array}{c}\text { CFCS V } \\
n=3\end{array}$ \\
\hline \multicolumn{6}{|l|}{ Sex } \\
\hline M & 9 & 3 & 3 & 2 & 2 \\
\hline $\mathrm{F}$ & 3 & 10 & 1 & 1 & 1 \\
\hline \multicolumn{6}{|l|}{ Age } \\
\hline Mean & 14.3 & 14.0 & 14.6 & \multirow{2}{*}{\multicolumn{2}{|c|}{$\begin{array}{c}15.6 \\
1.6\end{array}$}} \\
\hline SD & 1.3 & 1.9 & 3.6 & & \\
\hline Range & $12.3-17.0$ & $10.5-18.1$ & $9.7-18.1$ & \multicolumn{2}{|c|}{$14.3-17.9$} \\
\hline \multicolumn{6}{|c|}{ Coexisting neurological diagnosis, multiples represented } \\
\hline ASD & 3 & 1 & - & - & - \\
\hline ADHD & 6 & 1 & 1 & - & - \\
\hline $\mathrm{HL}$ & 1 & - & 1 & - & - \\
\hline SD & 2 & 4 & 2 & 1 & 3 \\
\hline CVI & - & - & - & - & 2 \\
\hline
\end{tabular}

\section{GMFCS}

$\begin{array}{llllll}\text { I } & 5 & 3 & 1 & - & - \\ \text { II } & 5 & 4 & 1 & - & - \\ \text { III } & 1 & 4 & - & - & 1 \\ \text { IV } & - & 2 & 2 & - & - \\ \text { V } & 1 & - & - & 3 & 2\end{array}$

MACS

$\begin{array}{llllll}\text { I } & 4 & 3 & - & - & - \\ \text { II } & 6 & 8 & 1 & - & - \\ \text { III } & 1 & 2 & 2 & - & - \\ \text { IV } & 1 & - & 1 & 1 & 1 \\ \text { V } & - & - & - & 2 & 2\end{array}$

\section{Cerebral palsy type}

Spastic

\section{Diplegia}

Hemiplegia (left)

Hemiplegia (right)

Triplegia

Quadriplegia

Ataxic

Mixed

Hypotonic

Unknown

$\begin{array}{llll}3 & - & - & - \\ 2 & - & - & - \\ 1 & 1 & - & - \\ 1 & - & - & - \\ 1 & 2 & - & 2 \\ 1 & 1 & 2 & - \\ 1 & - & - & - \\ - & - & - & - \\ 3 & - & 1 & 1\end{array}$

hearing loss, SD: seizure disorder, CVI: cortical visual impairment

Note: GMFCS refers to the Gross Motor Classification System level (Palisano et al., 1997); MACS refers to Manual Ability Classification System (Eliasson, Krumlinde-Sundholm, Rosblad, et al., 2006) 
Thirty-five participants with CP (19 males) participated in the current project. Table 2 shows the demographic characteristics of participants in this study. The overall mean age was 14.5 years (range: 9-18 years; SD: 1.9), and grade levels in school ranged from $4^{\text {th }}-12^{\text {th }}$. Most participants in this project attended a school in Wisconsin $(n=29)$, while others attended schools in neighboring states of Minnesota $(n=2)$ or Illinois $(n=4)$. There were 30 different school districts involved in the provision of IEP services for participants in this study. Six participants attended school in the same district, and three of these participants attended the same school. We were not able to ascertain how many different SLPs were involved in the creation of IEP goals; however, the three participants who attended the same school likely also had the same SLP. Thirty of the IEPs collected were active at the time of the participant's in-person visit, while five had implementation dates starting after the in-person visit. The start dates of five IEPs that had implementation dates after data collection ranged from one week to seven months after the in-person diagnostic session.

\section{Procedures}

Data were gathered using two sources: 1) an in-person, diagnostic session with each participant and 2) transcription and coding of each participant's IEP goals.

\section{Diagnostic Sessions}

Data collection involved one in-person visit to our laboratory. Participants completed a battery of assessments to characterize their speech, language, and communication strengths and challenges. A research SLP facilitated the assessments. In-person visits lasted approximately 2.5 hours with intermittent breaks. During direct assessment, parents of participants simultaneously completed questionnaires about their child's communication and behavior in a separate room. Methods used in obtaining measures of receptive language, cognition, EF, speech intelligibility, and functional communication from each in-person visit are explained below:

Receptive language via standardized assessment: One of three standardized receptive language assessments was administered to each participant based on age and level of motor impairment. Assessments were the Test for Reception of Grammar (TROG-2, $n=23$ ) (Bishop, 2003), which evaluates understanding of grammatical constructions within sentences; the Test for Auditory Comprehension of Language (TACL-4, $n=3$ ) (Carrow-Woolfolk, 1999), which assesses understanding of single-word vocabulary, grammatical morphemes and elaborated phrases; and the Peabody Picture Vocabulary Test (PPVT-4, $n=4$ ) (Dunn \& Dunn, 2007), which is very limited in its scope, assessing only understanding of single-word vocabulary. Five participants were unable to complete a receptive language assessment due to motor and/or visual impairment. Three different tests were used due to differences in age and motor ability of the participants. All participants were enrolled in a larger longitudinal study of speech and language development. That protocol dictated the use of the TACL-4 through the age of 12;11, and the TROG-2 from 13;0 - 16 years of age. Children who could not tolerate the longer and more complex tests (TACL and TROG), were administered the more basic PPVT-4. All three standardized measures yield raw scores that are converted to standardized scores with a mean of 100 and standard deviation of 15. Participants were categorized as having language comprehension impairment if their standard score was 1.5 standard deviations or more below the mean value per the technical manual. 
Non-verbal cognition via standardized assessment: The Brief IQ screener of the Leiter-R (Roid \& Miller, 1997) was administered to participants; nine were unable to complete testing due to severity of motor impairment and/or impaired vision. The Brief IQ screener consists of four subtests that assess visualization and reasoning skills through presentation of visual stimuli without the use of language. Examples of non-verbal cognition skills that are accessed during testing include pattern detection and sequencing, identification of a visual stimuli within a visually complex display, and mental manipulation and rotation of fractured images. The assessment yields raw scores that are converted to a standardized composite score with a mean value of 100 and standard deviation of 15. Participants were categorized as having a non-verbal cognitive deficit if their score was two standard deviations or more below the mean value, per the test manual.

EF via parent questionnaire: The Behavior Rating Inventory of Executive Function (BRIEF-2) (Gioia et al., 2015) is a parent rating measure that evaluates a child's behavior within the context of nine EF domains: inhibit, self-monitor, shift, emotional control, initiate, working memory, plan/organize, task-monitor, and organization of materials. Parents respond to 63 behavior-based statements while thinking of their child's current characteristics in these nine domains, and their responses generated a Global Executive Composite (GEC) T-score. T--scores have a mean value of 50 and a standard deviation of 10. Participants were categorized as impaired in EF skills if they received a GEC T-score that exceeded 1.5 standard deviations from the mean value per the manual. Parents did not complete the BRIEF-2 or data were incomplete for nine participants.

Speech severity and functional communication via clinician ratings: Two clinician-rating tools were utilized to classify communication skills of the participants in this study, the Viking Speech Scale (VSS) (Pennington et al., 2013) and the CFCS (Hidecker et al., 2011). The VSS is a clinician-rating tool developed for individuals with CP that indicates the presence and severity of speech motor impairment (Pennington et al., 2013). It uses four ordinal levels that range from I, "Speech is not affected by motor disorder" to IV, "No understandable speech". Definitions of each level are shown in Table 3. We also used the previously described CFCS (Hidecker et al., 2011) to classify functional communication (see Table 1).

Table 3. Viking Speech Scale levels for children with CP per Pennington et al., 2013.

\begin{tabular}{ll}
\hline Level & Definition \\
\hline I & Speech is not affected by motor disorder. \\
II & Speech is imprecise but usually understandable to unfamiliar listeners. \\
III & Speech is unclear and not usually understandable to unfamiliar listeners out of context. \\
IV & No understandable speech. \\
\hline
\end{tabular}

Following each in-person visit, the SLP immediately rated the participant using the published guidelines of the VSS and CFCS. An additional SLP rater who did not participate in the diagnostic session made a second, independent rating of VSS and CFCS levels for each participant. Ratings were completed based on a review of approximately fifteen minutes of video which included a parentchild interaction (familiar communication partner) and casual conversation with the clinician (unfamiliar communication partner). Following initial ratings, the SLPs met to review any non-agreeing ratings and ultimately placed each participant into a final level for both the VSS and the CFCS. Consensus was obtained through joint viewing of the participant's video recording. We used weighted Kappa statistics, as recommended for ordinal data (Ranganathan et al., 2017), to evaluate inter-rater agreement. This measure has an advantage over traditional unweighted Kappa values in that it reflects the distance of 
ordinal disagreements from each other, thus accounting for adjacent ratings between two individuals differently than non-adjacent ratings.

For the VSS, interrater agreement between the two SLP raters was $83.33 \%$ (weighted kappa = 0.90), which indicated strong agreement (McHugh, 2012). Of the six participants with non-agreeing VSS ratings, all six were within a one-level classification difference (e.g., first rater assigned a participant to level II, while second rater assigned the same participant to level III).

For the CFCS, interrater agreement between the two raters was 50\% (weighted kappa $=0.72$ ), which indicated moderate agreement per McHugh (2012). Of the 18 participants with non-agreeing CFCS ratings, 14 were within a one level classification difference (e.g., first rater assigned a participant to level II, while second rater assigned the same participant to level III). These findings are consistent with other published studies examining the CFCS (Hidecker et al., 2011; Hustad et al., 2016b; Palisano et al., 2018). Note, however, that reliability concerns for the CFCS are mitigated in the present study by our use of consensus procedures for resolving classification differences to come to a collaborative decision.

\section{IEP Transcription and Coding Procedure}

To examine speech and language school-based services, each student's IEP was reviewed, coded, and analyzed using a similar procedure to Hustad and Miles (2010), which involved six steps as follows.

1. All goal statements listed on the student's IEP were located and transcribed, verbatim, into a secure online database. Goal statements included long-term goal language, as well as short-term objectives.

2. Using node assignments with NVivo software (Version 12.6; QSR International Pty Ltd., 2019), all goal statements were aggregated across participants and reviewed by a first rater to identify common themes within goal language. The first rater considered ASHA's Scope of Practice in Speech Pathology, Speech-Language Pathology Service Delivery Areas (ASHA, 2016) in determining categories for goal statements.

3. From the aggregate pool of goals obtained from participants' IEPs, seven target skill categories were identified and named based on similarities within goal statements. These were: augmentative and alternative communication (AAC), EF, hearing, speech, language, literacy, and non-speech and language. We did not include goals that were coded as "non-speech and language" in further analyses, beyond coding reliability. Operational definitions were created for each target skill category and can be found in the supplemental material for this paper. Early iterations of the goal sorting stage included the additional target categories of speech sound production, speech subsystems, and intelligibility to differentiate speech-related interventions; however, very few participants had treatment programs in these narrower categories-likely because participants included in the current study were older and these goals were no longer appropriate. Therefore, these categories were collapsed into a single "speech" category.

4. All speech and language goal statements were examined further by the first rater, and a data reduction process was employed. Goal statements were broken down into a further unit of analysis deemed a unique measurable treatment unit (UMTU). UMTUs are defined as a segment of a goal 
that is both measurable and only addresses one target skill area. The decision to break goal statements into smaller units of meaning was made when the first rater observed differences in how practicing SLPs wrote IEPs goals. For example, some goals found in participants' IEPs were written to address one target skill: "With modeling and support, child will follow the behavior plan when he is unable to de-escalate and problem-solve and fill out his reflection form when leaving the classroom $50 \%$ of the time in the IEP term." This goal statement is measurable for level of attainment (50\%) and addresses only one target skill category (EF). Therefore, the entire goal is composed of only one UMTU and is relatively straightforward for classification into a single target skill category. Conversely, other goals in participants' IEPs were written to address multiple target skills: "Child will improve her communication skills by meeting 2 of 2 objectives below: obj 1: correct misarticulations of the $/ \mathrm{k} /$ and $/ \mathrm{g} /$ sound in short responses in 3 out of five opportunities; obj 2: identify 2 items that belong in a given category and identify the category given a list of related items with $75 \%$ accuracy". This goal statement has multiple measurable objectives that belong to different target skill categories (i.e., objective 1-speech, objective 2-language). The UMTU coding strategy was employed to ensure that each meaningful piece of a goal statement that was measurable and unique was credited toward specific target skills.

5. Using the operational definitions for seven target skill categories and a guideline for reducing goal statements into smaller UMTU segments, a second rater was trained to determine how many UMTUs existed for all IEP goals and instructed to assign each identified UMTU to a target skill category. Inter-rater reliability was calculated using NVivo's coding comparison query which analyzes the percentage of agreement between two raters for whether a presented text is assigned to a node. The coding comparison query also provides a Cohen's Kappa coefficient to factor in chance agreement between coders. Across all seven target skill areas, the overall kappa value was 0.89 ( $97.69 \%$ agreement), indicating strong inter-rater reliability between the two coders. Inter-rater values for each of the seven target skill categories are represented in Table 4.

Table 4. Inter-rater reliability measures for coding of IEP goals.

\begin{tabular}{lccc}
\hline $\begin{array}{l}\text { Target Skill / Node } \\
\text { Assignment }\end{array}$ & Agreement (\%) & $\begin{array}{c}\text { Cohen's } \\
\text { Kappa Coefficient }\end{array}$ & $\begin{array}{c}\text { Interpretation } \\
\text { McHugh (2012) }\end{array}$ \\
\hline AAC & 96.43 & 0.82 & Strong \\
Executive Functioning & 96.58 & 0.77 & Moderate \\
Hearing & 100.00 & 0.99 & Almost perfect \\
Speech & 100.00 & 1.00 & Almost perfect \\
Language & 97.62 & 0.86 & Strong \\
Literacy & 96.76 & 0.86 & Strong \\
Non-Speech and Language & 96.41 & 0.92 & Almost perfect \\
\hline Total Agreement Ratings & $\mathbf{9 7 . 6 9}$ & $\mathbf{0 . 8 9}$ & Strong \\
\hline
\end{tabular}

Note: Interpretation of Cohen's kappa per McHugh (2012).

6. The first and second raters compared differences in coding and discussed each difference in UMTU and/or target skill category placement. Consensus was used to make final coding determinations for target skill category placement; operational definitions of target skill areas were updated as needed. 


\section{Analyses}

Because of the small number of participants in this study and more specifically within individual CFCS levels, we used non-parametric statistical tests. We addressed both of our research questions using the Jonckheere-Terpstra test for ordinal variables (Ali et al., 2015), which allowed us to examine whether the number of deficit areas differed among the CFCS levels for Question 1, and whether the number of speech and language treatment goal areas differed among the CFCS levels for Question 2. For Question 1, we also used Kruskal-Wallis tests with Mann-Whitney U follow-up contrasts to examine whether there were differences among mean scores across the four CFCS levels within each deficit area. Follow-up tests, where appropriate, were adjusted for multiple comparisons using the Hochberg correction (Hochberg, 1988). Analyses were carried out in SPSS for Macintosh, Version 26.0 .

Note that for both research questions, we used four levels for the CFCS. We grouped level IV and level $\mathrm{V}$ together due to very small numbers of participants in each group. Further, CFCS level IV-V could not be included in statistical analyses for Question 1 because of missing data associated with difficulty completing standardized tests that required manual manipulation and pointing to indicate responses. All participants in CFCS level IV-V had VSS ratings of 4, indicating that these individuals were unable to produce speech. This group is included in results for Question 2.

\section{Results}

\section{Underlying Impairment Profiles by CFCS}

Table 5 shows speech, language, and cognitive ratings and scores by CFCS level. Results generally suggested that VSS scores, indexing severity of speech impairment, were worse for participants in more involved CFCS levels (more challenges with functional communication). Inferential statistics indicated a significant omnibus result for VSS among groups $\left(\chi^{2}(3)=27.96 ; p<.001\right)$. Follow up contrasts revealed significant differences between CFCS I vs. II $(U=22.0, p<.001)$, CFCS I vs. III $(U=0, p<.001)$, CFCS I vs. IV $(U=0, p<.001)$, CFCS II vs. III $(U=4.5, p=.006)$, and CFCS II vs. IV $(U=0, p<.001)$, confirming that participants in less involved CFCS levels had better speech motor abilities than those in more involved levels. The only exception was that the difference in VSS for CFCS III vs. IV was not significant.

Non-verbal cognitive skills and receptive language appeared more impaired for those in more involved CFCS levels (greater functional communication limitations). Inferential statistics indicated a significant omnibus result for non-verbal cognition among groups $\left(\chi^{2}(2)=6.350 ; p=.042\right)$. Follow up contrasts revealed significant differences between CFCS I vs. II $(U=21.5, p=.007)$, and CFCS I vs. III $(U=2, p=.028)$, confirming that those in the least involved CFCS level had better non-verbal cognitive skills than those in more involved groups. However, for receptive language, the omnibus test was not significant, indicating no significant differences among levels of the CFCS.

EF deficits appeared to be greater in participants in the least involved CFCS level (better functional communication), as indicated by higher scores on the BRIEF-2. Inferential statistics indicated a significant omnibus result $\left(\chi^{2}(2)=9.171 ; p=.010\right)$. Follow up contrasts revealed a significant difference only for CFCS I vs. II $(U=32.0, p=.021)$, indicating that EF difficulties were greater in the less involved CFCS level. 
Table 5. Underlying deficits scoring summary for children with CP by CFCS leveling

\begin{tabular}{|c|c|c|c|c|}
\hline & $\begin{array}{l}\text { CFCS I } \\
(n=12)\end{array}$ & $\begin{array}{l}\text { CFCS II } \\
(n=13)\end{array}$ & $\begin{array}{c}\text { CFCS III } \\
(n=4)\end{array}$ & $\begin{array}{c}\text { CFCS IV and V } \\
(n=6)\end{array}$ \\
\hline \multicolumn{5}{|l|}{ Viking Speech Scale } \\
\hline Rating I & 1 & - & - & - \\
\hline Rating II & 11 & 4 & - & - \\
\hline Rating III & - & 8 & 2 & - \\
\hline Rating IV & - & 1 & 2 & 6 \\
\hline \multicolumn{5}{|l|}{ Non-verbal Cognition } \\
\hline SS Range & $38-117$ & $36-95^{\star 1}$ & $36-56^{* 2}$ & $-{ }_{-}^{*} 6$ \\
\hline SS Mean & 72.83 & $50^{\star 1}$ & $46^{* 2}$ & $-{ }^{*} 6$ \\
\hline SS Std. Deviation & 22.91 & $6.93^{* 1}$ & $10^{*} 2$ & $-{ }^{*} 6$ \\
\hline$\%$ of group w/ deficit & $58 \%$ & $92 \%{ }^{* 1}$ & - & - \\
\hline \multicolumn{5}{|l|}{ Executive Functioning (EF) } \\
\hline GEC T score Range & $56-90^{* 2}$ & $41-78$ & $50-57^{* 1}$ & $-{ }_{-}^{*} 6$ \\
\hline GEC T score Mean & $73^{* 2}$ & 58.46 & $55^{* 1}$ & $-{ }^{*} 6$ \\
\hline GEC T score Std. Deviation & $9.88^{* 2}$ & 11.33 & $3.56^{* 1}$ & $-{ }^{*} 6$ \\
\hline$\%$ of group $w /$ deficit & $90 \%{ }^{*} 2$ & $23 \%$ & - & - \\
\hline \multicolumn{5}{|l|}{ Receptive Language } \\
\hline SS Range & $55-111$ & $43-104$ & $20-90$ & $-{ }_{-}^{*} 5$ \\
\hline SS Mean & 81.42 & 67.92 & 52.5 & $--^{* 5}$ \\
\hline SS Std. Deviation & 20.34 & 18.36 & 25.12 & $-{ }^{*} 5$ \\
\hline$\%$ of group $w /$ deficit & $50 \%$ & $62 \%$ & $75 \%$ & - \\
\hline \multicolumn{5}{|c|}{$\begin{array}{l}\text { Note: " } \text { number indicates \# of participants in CFCS level with missing data; SS = Standard Score; GEC = Global } \\
\text { Executive Composite }\end{array}$} \\
\hline \multicolumn{5}{|c|}{ Higher score for EF, obtained from the BRIEF, reflected increased deficits. } \\
\hline \multicolumn{5}{|c|}{$\begin{array}{l}\text { For children in CFCS IV and V, standardized scores were not available for measures of non-verbal cognition, EF, } \\
\text { and receptive language because of the severity of their motor involvement. For children in CFCS III, standard } \\
\text { scores for cognition were available for only two of four children. }\end{array}$} \\
\hline \multicolumn{5}{|c|}{$\begin{array}{l}\text { Table } 6 \text { shows the numbers of participants in each CFCS level by binary categories for } \\
\text { ice / absence of underlying speech, language, EF, and cognitive deficits. Figure } 1 \text { shows a tally } \\
\text { number of participants with each type of underlying impairment by CFCS level. Results suggest } \\
\text { dividuals in each CFCS level show a range of different deficits. Statistical results indicated that } \\
\text { mber of total deficit areas was not significantly different among CFCS levels, with the average } \\
\text { r of deficit areas per level being } 3 \text { for CFCS I, II, and III. }\end{array}$} \\
\hline
\end{tabular}


Table 6. Binary ratings of presence / absence of underlying deficits in each speech and language domain by CFCS level.

\begin{tabular}{lcccc}
\hline & $\begin{array}{c}\text { CFCS I } \\
(\mathbf{n = 1 2})\end{array}$ & $\begin{array}{c}\text { CFCS II } \\
(\mathbf{n}=\mathbf{1 3})\end{array}$ & $\begin{array}{l}\text { CFCS III } \\
(\mathbf{n}=\mathbf{4})\end{array}$ & $\begin{array}{l}\text { CFCS IV-V } \\
(\mathbf{n}=\mathbf{6})\end{array}$ \\
\hline Speech Deficit & 11 & 13 & 4 & 6 \\
Yes & 1 & 0 & 0 & 0 \\
No & - & - & - & - \\
Missing Data & & & & \\
Receptive Language Deficit & 6 & 8 & 3 & 1 \\
Yes & 6 & 5 & 1 & 0 \\
No & - & - & - & 5 \\
Missing Data & & & & \\
Non-verbal Cognition Deficit & 7 & 11 & 2 & 0 \\
Yes & 5 & 1 & 0 & 0 \\
No & - & 1 & 2 & 6 \\
Missing Data & & & & \\
Executive Functioning (EF) Deficit & & 13 & 0 & 0 \\
Yes & 9 & 0 & 3 & 6 \\
No & 1 & - & 1 & \\
Missing Data & 2 & & & \\
\hline
\end{tabular}

Note: Speech deficits were defined as Viking Speech Scale ratings greater than I. Receptive language deficits were defined as standardized test scores that were 1.5 or more standard deviations below the mean. Non-verbal cognitive deficits were defined as standardized test scores that were two or more standard deviations below the mean. EF deficits were based on standardized test scores that were 1.5 or more standard deviations above the mean. Criteria were based on published test instructions for each measure.

Figure 1. Number of children with underlying deficits in EF, nonverbal cognition, receptive language, or speech by CFCS level. Note that 35 children were included in the study. The number of children with underlying deficits in each area is not mutually exclusive; thus, a child could have deficits within all four areas or only one area. The black line in each panel represents the total number of children with data in each group.
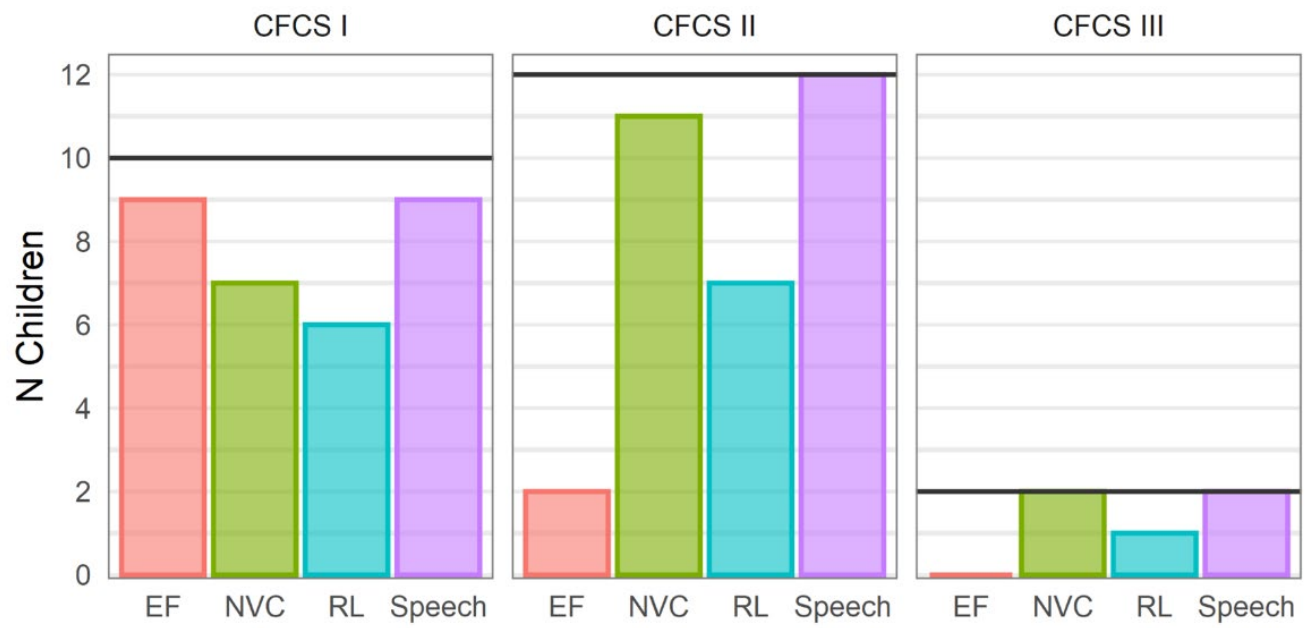

Deficit Abbreviations: EF = executive functioning, NVC = non-verbal cognition, $\mathrm{RL}=$ receptive language.

Note: Figure 1 includes only children with complete assessment data for all four deficit areas. Children in CFCS IV-V ( $n=6$ ) did not have data for EF, NVC, or RL, though all children in that group had speech deficits. For CFCS III, only 2 of 4 children had data for EF, NVC, and RL. We highlight that all children in CFCS III had speech deficits $(\mathrm{N}=4)$. 


\section{School-Based IEP Goals by CFCS}

Table 7 shows speech and language goals by CFCS level. Figure 2 illustrates the number of participants with goals in each of the six areas by CFCS level. Results indicated that 4-6 unique goals (UMTUs) were targeted on average for participants who had IEPs, regardless of CFCS level. Goal areas targeted for intervention overlapped considerably across CFCS levels, with at least four different goal areas represented for CFCS levels I, II, and III. CFCS level IV-V had two goal areas represented. The only goal area that was represented across all CFCS levels was literacy. In addition, those in more severe CFCS levels (i.e., IV-V) tended to be more likely to have AAC goals. Statistical results indicated that the number of goal areas was not significantly different among CFCS levels. We also examined speech and language goals by impairment profiles within CFCS levels, as shown in Table 8. A descriptive summary of findings regarding how impairment profiles mapped onto speech and language goal areas for each CFCS level is provided below.

Table 7. Speech and language IEP goal breakdown by CFCS level.

\begin{tabular}{|c|c|c|c|c|}
\hline & $\begin{array}{l}\text { CFCS I } \\
(n=12)\end{array}$ & $\begin{array}{l}\text { CFCS II } \\
(n=13)\end{array}$ & $\begin{array}{l}\text { CFCS III } \\
(n=4)\end{array}$ & $\begin{array}{l}\text { CFCS IV and V } \\
(n=6)\end{array}$ \\
\hline \multicolumn{5}{|c|}{ Unique Measurable SLP Units } \\
\hline UMTU range & $1-13$ & $1-11$ & $4-9$ & $3-8$ \\
\hline UMTU mean & 4.33 & 4.62 & 6 & 5.17 \\
\hline UMTU Std. Deviation & 3.04 & 2.92 & 2.12 & 1.77 \\
\hline \multicolumn{5}{|c|}{ Target Skill Areas - percentage of children w/ at least one UMTU } \\
\hline AAC & $0 \%$ & $8 \%(1 / 13)$ & $75 \%(3 / 4)$ & $100 \%(6 / 6)$ \\
\hline Executive Functioning (EF) & $83 \%(10 / 12)$ & $62 \%(8 / 13)$ & $0 \%$ & $0 \%$ \\
\hline Hearing & $8 \%(1 / 12)$ & $0 \%$ & $0 \%$ & $0 \%$ \\
\hline Speech & $0 \%$ & $23 \%(3 / 13)$ & $2(1 / 4)$ & $0 \%$ \\
\hline Language & $42 \%(5 / 12)$ & $77 \%(10 / 13)$ & $25 \%(1 / 4)$ & $0 \%$ \\
\hline Literacy & $75 \%(9 / 12)$ & $69 \%(9 / 13)$ & $75 \%(3 / 4)$ & $17 \%(1 / 6)$ \\
\hline
\end{tabular}

Note: UMTU stands for "unique measurable treatment unit" and is the product of reducing lengthy IEP goal statements into smaller units that are measurable and only address one target skill area. Each UMTU is only assigned to one of six mutually exclusive target skill categories.

Figure 2. Number of children with targeted speech and language goal areas according to IEPs by CFCS level. Note that 35 children were included in the study. The number of children with goals in each area is not mutually exclusive; thus, a child could have goals in multiple areas, or only one area. The black line in each panel represents the total number of children with data in each group.

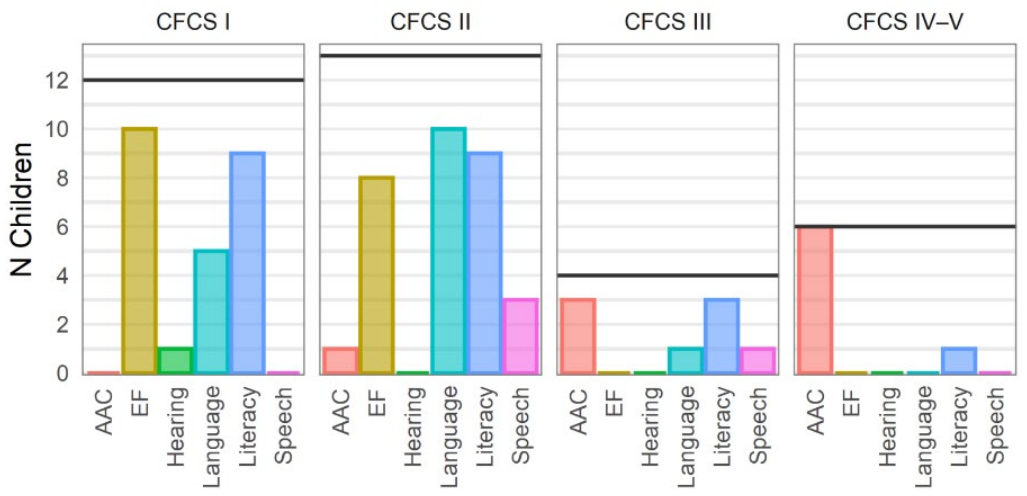

Abbreviations: $A A C=$ augmentative and alternative communication, $E F=$ executive functioning.

Note: Figure 2 includes all children in this study, regardless of whether data were missing for underlying impairments. 
For CFCS I $(n=12)$, there were six different combinations of impairment profiles (see Table 8), which varied widely from a single deficit in one area (speech) to deficits in all four assessed areas. The most common profile involved deficits in all four areas assessed for this study (speech, receptive language, EF, and non-verbal cognition) $(n=5)$. Among the collective 12 participants in this group, the two most common targeted areas for intervention were EF and literacy. Nine participants in CFCS I showed deficits in EF skills, and eight of those nine individuals were treated with at least one EF goal in school. In addition, two individuals who did not show deficits in EF had an EF goal in school. Across impairment profiles in CFCS I, nine received literacy-based intervention; literacy deficits were not assessed in this study, so we are unable to evaluate mapping of deficits to goals for this area. For receptive language abilities, five of six participants who had an underlying deficit had a corresponding goal in language skills. Eleven of twelve participants had a mild speech deficit (VSS rating II "speech is imprecise but usually understandable to unfamiliar listeners"); however, none of the eleven had a speech goal or had an AAC system.

For CFCS II $(n=13)$, there were again six different combinations of impairment profiles (see Table 8). As with CFCS I, impairment profiles varied from a single deficit in one area (speech), to deficits in all four assessed areas. In contrast to findings from CFCS I, the most common impairment profile involved co-occurring deficits in speech, receptive language, and non-verbal cognition $(n=6)$. The most common targeted areas for intervention were EF, literacy, and language. All participants in this group with an EF deficit $(n=3)$ had a corresponding EF intervention goal, and five participants who did not show deficits in EF were also treated in this goal area. All participants with a receptive language deficit had a language-based intervention goal, and two in this group who did not show deficits in receptive language had a targeted language goal. [Note that both participants had goals focusing on pragmatic language.] Nine participants received literacy-based intervention. Regarding speech, all thirteen participants in CFCS II had a deficit in speech production, as defined by a VSS score of II, III, or IV. Of these, nine received a VSS score of III or IV, indicating that speech is not usually understandable to unfamiliar listeners out of context or is not understandable at all. Yet, only four of nine had goals that addressed speech production; and only one had AAC goals. Collectively, fewer than half of those with severe speech deficits in this group had a corresponding intervention goal to improve or supplement their spoken communication. Only one child in this group had an AAC system in place, which was a high-tech app-based system on an iPhone.

For CFCS III $(n=4)$, there were three different combinations of impairment profiles. Note, however, that this group had only four participants. All participants in this group had at lea st two deficit areas that included both speech and receptive language or non-verbal cognitive abilities. The major areas of intervention for this group were AAC and literacy. Three of four participants in this group had a literacy intervention goal, and of the three with receptive language deficits, only one received a corresponding language-based intervention goal. All four participants had deficits in speech; three had intervention goals in AAC, while the fourth child had a speech production goal. Three participants in this group had AAC systems in place, and all were high tech (e.g., Tobii Dynavox with eye gaze, TouchChat on iPad).

For CFCS IV-V $(n=6)$, there were two impairment profiles observed. Note, however, that these individuals were not able to complete cognitive and language tests because of severe motor limitations and / or vision limitations. Thus, it was difficult to examine how deficit areas mapped onto school-based goals for this group. However, all six had a VSS score of IV, "no understandable 
speech", and all six had an AAC based intervention goal in their IEP. One of the six had a literacybased intervention program distinct from AAC intervention. Four participants in this group had AAC systems in place; one was high tech (i.e., Tobii Dynavox with eye gaze); three were low/mid tech (e.g., switches and communication boards).

Table 8. Speech and language goals by CFCS level and deficit profile.

\begin{tabular}{|c|c|c|c|c|c|c|}
\hline & AAC & EF & Hearing & Speech & Language & Literacy \\
\hline \multicolumn{7}{|l|}{ CFCS I $(n=12)$} \\
\hline$S(n=1)$ & - & 1 & - & - & - & - \\
\hline$S+R L(n=1)$ & - & 1 & - & - & 1 & 1 \\
\hline$S+E F(n=3)$ & - & 3 & - & - & - & 1 \\
\hline$S+N V C(n=1)$ & - & - & - & - & - & 1 \\
\hline$N V C+E F(n=1)$ & - & 1 & - & - & - & 1 \\
\hline$S+R L+E F+N V C(n=5)$ & - & 4 & 1 & - & 4 & 5 \\
\hline Total & 0 & 10 & 1 & 0 & 5 & 9 \\
\hline \multicolumn{7}{|l|}{ CFCS II $(n=13)$} \\
\hline$S(n=1)$ & - & 1 & - & - & - & - \\
\hline$S+N V C(n=3)$ & 1 & 1 & - & - & 1 & 2 \\
\hline$S+R L+E F(n=1)^{*}$ & - & 1 & - & 1 & 1 & 1 \\
\hline$S+R L+N V C(n=6)$ & - & 3 & - & 2 & 6 & 5 \\
\hline$S+E F+N V C(n=1)$ & - & 1 & - & - & 1 & - \\
\hline$S, R L, E F, N V C(n=1)$ & - & 1 & - & - & 1 & 1 \\
\hline Total & 1 & 8 & 0 & 3 & 10 & 9 \\
\hline \multicolumn{7}{|l|}{ CFCS III $(n=4)$} \\
\hline$S+R L(n=2)^{*}$ & 2 & - & - & - & - & 2 \\
\hline$S+N V C(n=1)$ & - & - & - & 1 & - & 1 \\
\hline$S+R L+N V C(n=1)$ & 1 & - & - & - & 1 & - \\
\hline Total & 3 & 0 & 0 & 1 & 1 & 3 \\
\hline \multicolumn{7}{|l|}{ CFCS IV-V (n=6) } \\
\hline$S(n=5)^{*}$ & 5 & - & - & - & - & 1 \\
\hline$S+R L(n=1)^{*}$ & 1 & - & - & - & - & \\
\hline Total & 6 & 0 & 0 & 0 & 0 & 1 \\
\hline
\end{tabular}

Target Skill Abbreviations: $A A C=$ augmentative and alternative communication, EF = executive functioning

Note: individual cell value indicates number of children with at least one targeted skill in the respective categories

Note: *indicates that number includes some children who did not complete all four assessments, therefore comprehensive underlying deficit profile is unknown

\section{Discussion}

Individuals with $\mathrm{CP}$ can have a wide range of communication strengths, challenges, and needs; they frequently require speech and language intervention in the school setting. In this study, we sought to examine associations between functional communication, underlying abilities in speech, cognition, EF, and language, and how individuals were being served with regard to their speech, language, and communication goals in school. Key questions focused on 1) underlying impairment profiles associated with different functional communication abilities; and 2) the types of speech and language goals that were targeted in school for those with different functional abilities and underlying impairments. There were several key findings. First, we found speech, non-verbal cognition, and receptive language skills were more impaired for individuals in higher CFCS levels (reflecting greater 
limitations in functional communication abilities). However, individuals at each CFCS level showed a range of impairments, with no significant differences among CFCS levels in the total number of areas of impairment. We also observed a range of goal types identified in the IEP for participants across all CFCS levels and impairment profiles. The only goal type addressed across all CFCS levels was literacy. These outcomes are discussed in subsequent sections, below.

\section{Underlying Impairment Profiles and Functional Communication Abilities}

Our first set of questions asked: For each level of the CFCS, what are the associated underlying deficits in speech, cognition, EF, and receptive language? Are there differences in patterns of underlying impairments observed in individuals with different functional communication profiles? We hypothesized that individuals within each CFCS level would have a range of underlying abilities in speech, cognition, EF, and receptive language, owing to the fact that different constellations of deficits can lead to similar functional communication profiles. In general, we found that those in CFCS levels associated with more restricted functional abilities also had impairments that were more severe in terms of magnitude, but the number of different impairment areas did not differ among CFCS levels.

For speech, our findings were straightforward and demonstrated that individuals with more severe speech impairment (as measured by VSS ratings) had more restricted functional communication abilities (as indexed by higher CFCS levels). This is consistent with previous studies showing strong associations between speech impairment and participation outcomes (McCormack et al., 2010; McCormack et al., 2009; Mei et al., 2014), speech impairment and functional communication using the CFCS (Coleman et al., 2015; Hidecker et al., 2018; Pennington et al., 2020) and speech impairment and functional communication using other measures of communication (Nathan, 2002; Virella et al., 2016). The finding that VSS scores indicative of more severe speech impairment occurred more frequently in CFCS levels associated with more restricted functional communication abilities is not surprising given that CFCS ratings are based on the effectiveness of a sender's ability to transmit a message alongside their ability to understand a message. When an individual has more difficulty producing intelligible speech, an overall decrease in comprehensibility of the sender's message would be expected without the use of other augmented communication modalities.

With regard to non-verbal cognitive abilities, results of the present study seem to suggest that participants in more involved CFCS levels tended to have greater cognitive deficits. However, we were not able to examine cognitive abilities among those with the most restricted functional communication (i.e., CFCS IV-V) because those individuals were unable to reliably complete cognitive testing. Previous studies have shown positive correlations between functional communication using the CFCS and intellectual function using measures of IQ (Choi et al., 2018; Compagnone et al., 2014); and our results are generally consistent with previous studies. However, research using alternative testing modalities that do not require motor skills is needed to better understand the cognitive abilities of the most severely involved individuals with CP, who are also the most likely to be in CFCS levels IV$\mathrm{V}$.

Receptive language skills did not differ among CFCS levels, indicating that individuals with a range of receptive language abilities were in each CFCS level. This finding is consistent with other studies (Choi et al., 2018; Hustad et al., 2016a). It is important to note that three different receptive language tests were used because there is no single standardized test that can accommodate speech and motor abilities across all levels of severity and age, and because of limitations associated with the 
larger longitudinal study from which the participants in the present study were recruited. We highlight that five children were unable to complete testing due to their severity levels. A large majority (23 out of 30 remaining participants) were able to complete the TROG-2; and 3 additional children completed the TACL, both of which evaluate language comprehension at the sentence-level. Four children completed the PPVT. Children who completed the latter two measures did not differ from the larger group and represented all CFCS levels. The necessity to use different assessments in the present study highlights a critical need for an accessible, high-quality standardized language assessment that allows children to use multiple communication modalities to indicate knowledge. Such measures are currently in development in Europe and Canada and are being translated into English (Geytenbeek et al., 2014).

Another interesting finding was the discrepancy between non-verbal cognitive findings and language comprehension findings in the present study. Previous work has suggested that these two measures tend to be consistent within participants (Soriano \& Hustad, 2021), thus we might expect to see the same pattern of results for language comprehension as for non-verbal cognition. One explanation in the present study is that not all individuals who were able to complete language comprehension testing, which was more amenable to adaptation for motor impairment, were able to complete cognitive testing. Thus, analyses of cognitive results are based on fewer participants.

Further, individual performance may have been less stable on cognitive measures than on receptive language measures because of greater motor demands for testing. In addition, in the present study language comprehension measures were obtained from among three different tests, depending on the age and abilities of the participant. Although we used standard scores to equate the different measures, this may have introduced an additional source of variability into the results of this study. Studies are needed to further examine the mutual and differential effects of receptive language and cognitive abilities in individuals with $\mathrm{CP}$ and their relation to functional communication abilities.

Our results for EF skills followed a different pattern than that for other impairment measures. Specifically, those in the least involved CFCS levels tended to have poorer EF skills than those in the more involved CFCS levels. Table 1 offers one potential perspective on this finding. Nine out of the 12 in CFCS I had a coexisting neurological diagnosis known to be associated with executive dysfunction, attention deficit disorder (6 participants) or autism spectrum disorder (3 participants). In contrast, only 2 of 13 in CFCS II and 1 of 4 in CFCS III had one of these conditions. These comorbidities are likely to have been a confounding factor in our findings, artificially elevating the proportion of individuals with EF deficits in CFCS I. Previous studies suggest that EF challenges tend to co-occur in children with different neurodevelopmental disabilities (Daunhauer et al., 2017; Mangeot et al., 2002) and in CP specifically (Sakash et al., 2018). Additional research is needed with a larger population to advance our understanding of how EF relates to functional communication abilities.

Lastly with respect to our first hypothesis, we found that the number of individual deficit areas was not significantly different among CFCS levels. Individuals in our sample had an average of three deficit areas across all CFCS levels included in this analysis (I, II, and III). Note that our data were incomplete for those in CFCS levels IV-V due to testing constraints, therefore this group was excluded from analysis. To examine number of deficit areas, we used binary ratings of the presence/absence of underlying deficits in each area (Table 6 ), without regard for the severity of deficits in each area. Our results indicated that there was not a consistent pattern of deficits within CFCS levels. In other words, not all individuals in CFCS I showed the same specific impairments and similarly, several different 
combinations of deficits were observed in CFCS I, II, and III. Overall, this suggests that underlying speech and language impairments associated with different CFCS levels in individuals with CP are variable.

The results of this study emphasize the idea that broad, subjective classification ratings of communication functioning such as the CFCS do not adequately indicate the complexities of an individual's underlying capacity in speech, cognition, and language. Conversely, measures evaluating underlying capacity in speech, cognition, and language are not indicative of functional communication abilities as revealed by the CFCS. Given the variability in strength and deficit profiles observed in individuals with $\mathrm{CP}$, it is possible to show impairments in all four areas examined in this study and also have communication skills that are effective enough to be classified as CFCS I. Similarly, different constellations of underlying deficit may manifest within the same CFCS level. Our study offers support for the notion that making assumptions about underlying deficit profiles and functional capabilities based on performance in a single area can be misleading, thus assessments should consider both impairment measures as well as indices of functional abilities. The same can be said for making assumptions on the areas of need requiring IEP goals for intervention, on which we provide additional perspective below.

\section{School-Based IEP Goals, Communication Abilities, and Impairment Profiles}

For our second set of research questions, we collected speech and language goal data from the IEPs of participants. We used a hybrid method involving qualitative coding of goals, which we then analyzed quantitatively. We asked the following questions: For each level of the CFCS, what types of speech and language goals and objectives are identified in the IEP? Are there differences among the types of goals and objectives targeted in school for individuals with different functional communication profiles? We hypothesized that a variety of underlying deficits within each CFCS level would lead to a diverse range of speech and language goals targeted in school, but that there would be a close match between goals targeted and underlying deficit areas.

Our results showed that participants had, on average, 4-6 unique speech/language treatment goals (averaged across different goal areas) in their IEP, with no significant differences in the number of goals being addressed across CFCS levels. Findings generally supported our hypothesis that there would be a range of goals targeted and a close match between goals targeted and underlying deficit areas across CFCS levels.

For participants in CFCS I, the most common profile had four deficit areas: speech, receptive language, EF, and nonverbal cognition. EF and literacy were the most common goal areas for participants in this level. Interestingly, 11 out of the 12 participants in CFCS I had speech deficits (VSS II for 11 children); however, none had speech goals. Given that participants in this group ranged in age from 12 to 17 years, that they had an underlying motor impairment, and that they had strong functional communication skills (based on the definition of CFCS I), it is unlikely that treatment addressing speech production would yield meaningful change, even though these participants did have speech deficits. As noted earlier, 9 participants in this group had EF challenges per our assessment, likely associated with co-morbidities of attention deficit disorder (ADD) or ASD; and 10 participants had at least one EF goal in the IEP. EF is a critically important ability for adolescents and is a multifaceted construct. Many youth could benefit from EF supports, and those with CP in particular are at risk for 
EF problems (Sakash et al., 2018). Therefore, proactive goals addressing EF are clearly beneficial for these individuals.

For those in CFCS II, the most common profile included deficits in three areas: speech, receptive language, and nonverbal cognition. The most common goal areas in this group were EF, literacy, and language. All of the individuals in CFCS II had some level of speech impairment—ranging from mild to severe ( 8 were VSS III, characterized by speech that is not usually intelligible to unfamiliar listeners out of context; 1 had no understandable speech)_but only 4 of 13 had speech goals and only 1 of 13 had AAC goals. In other words, fewer than half of individuals in CFCS II had an intervention goal focused on improving or supplementing spoken communication. Given that CFCS II is defined as "Effective but slower paced sender and/or receiver with unfamiliar and/or familiar partners," it seems likely that most individuals at this level were able to compensate for speech deficits and therefore did not require school based services to address functional communication related to AAC or speech. CFCS level II had the largest percentage of participants with at least one language goal ( $77 \%$; 10 of 13 participants); and 8 of 13 participants in this group had receptive language deficits per our direct assessment. We did not characterize expressive language abilities in this study, however, we expect that at least some individuals may have had expressive language problems independent of receptive abilities. Literacy was also addressed for 9 of 13 participants. We did not examine literacy skills and therefore cannot discuss how impairments in this area related to IEP goals; however, literacy is clearly a key focus in education and is therefore a critical area for SLPs to address. EF was also a frequently targeted goal area for CFCS II, with 8 of 13 participants (62\%) having at least one EF goal. However, only three in this group had EF deficits based on our assessment. One explanation for this discrepancy may relate to the measurement of EF. We used the BRIEF (Gioia et al., 2000), a standardized parent report tool. SLPs working in a school setting may be more sensitive to EF challenges in middle and high school students than a parent questionnaire, such as the BRIEF, because of the time they spend on a daily basis working with students in the complex school environment. One area for further study is the examination of other measures of EF that might provide deeper insights into EF to capture the kinds of clinical observations that SLPs use to identify EF goals for middle and high school students.

For participants in CFCS III, we observed three different deficit profiles, all including speech impairment. Again, it is important to note that this level includes a small sample $(n=4)$ and two were not able to complete nonverbal cognitive assessments. All four participants in this group had some form of speech involvement alongside either receptive language deficits $(n=2)$, cognitive deficits $(n=$ $1)$, or both $(n=1)$. Three participants had AAC goals, and three participants had literacy goals. It was surprising that AAC was not addressed for all four individuals in this level given that all were either VSS III or IV, indicating severe speech deficits and given that CFCS III is defined as being an effective communicator only with familiar partners. This finding is consistent with previous research suggesting that there may be a bias against use of AAC for those with any speech production capacity, even when intelligibility is markedly compromised (Hustad \& Miles, 2010). Results highlight a need to support SLPs in learning about the ways that AAC can be used in conjunction with speech or to support speech to enhance communication with unfamiliar communication partners.

For CFCS IV-V, all participants had speech deficits; and one had receptive language deficits as well (5 of 6 could not complete receptive testing, thus it is unknown whether more participants in this group had receptive language deficits). Note also that testing could not be completed for cognitive 
abilities and EF for individuals in this group. Therefore, we do not have a complete picture of overall ability and deficit profiles for this small sample. However, the primary focus of speech and language services for these participants was AAC, with all individuals (6 of 6 ) having AAC goals in the IEP. In addition, one also had literacy goals, but no other goal areas were addressed for this group. For those with the most impaired speech and the most restricted functional communication abilities, SLPs and the educational team were highly attuned to improving basic functional communication in the school setting.

\section{Limitations and Future Directions}

Despite our unique findings using a mixed-methods research design, several limitations exist in the present study. First, our sample comprised only 35 participants with CP who were heterogeneous in their age, grade level, and ability profile. Our analysis involved comparing a mix of standardized assessments and subjective ratings completed in our laboratory with a retrospective examination of speech and language goals included in legal IEP documents to understand how these individuals were being served in school. We were not able to validate the extent to which the stated goals and objectives in the IEP were actually being addressed, how they were being addressed, or whether other things were also being addressed. Furthermore, we did not examine the types of assessments completed by school staff used in the identification of deficit areas needing to be addressed. Additional research is needed in this population directly examining relations between school-based speech and language assessment, treatment, and outcomes with respect to communication functioning to fully understand the impacts of SLP services on the outcomes of individuals with CP. Research in this area may also be useful in identifying trends across CFCS levels for the provision of AAC and literacy services, as well as provider-level trends for identifying goal area "gaps" for the purposes of determining areas of need for continuing education.

All of the participants in our sample were from the upper Midwestern region of the US, and most were from Wisconsin. We do not know how school, or provider-level trends in service provision and regional trends in state-specific educational requirements might impact IEP goals and objectives for those with $\mathrm{CP}$, but this is an important area for future investigation.

We did not have literacy evaluation data for the participants in this study. Literacy was an important focus of educational goals and was the only goal area that touched all CFCS levels in some way. Future studies should examine literacy abilities, needs, and services for individuals with CP to support SLPs' participation in reading and writing intervention for individuals with complex communication needs. A related limitation is that we did not have expressive language evaluation data for our sample. Although the goal area "Language" included both receptive and expressive domains, assessment data on both receptive and expressive language abilities would have offered additional perspective on ability profiles.

We were not able to obtain impairment-level standardized test results for the most severely involved participants in this study because of motor and/or visual impairment ( 9 of 35 participants). These individuals comprised all of CFCS IV-V, and half of CFCS III. Alternative testing measures sensitive to reduced motor abilities are needed that allow us to understand the unique set of strengths and deficits of each individual. This information could play an important role in ensuring that students receive the most appropriate educational programming to advance their abilities. 
Finally, the analyses presented in this study from IEPs were based on qualitative coding of goal areas which were then analyzed quantitatively. Because there was considerable variability among IEPs with regard to how goals and objectives were written, we employed broad categories in our coding. As a result, many subtleties of skill areas being addressed may not be represented in this study. For example, because goals and objectives were coded in a mutually exclusive manner, a goal that was coded as "AAC" may have focused on using a communication system in a complex social interaction, which could also be considered a pragmatic language goal. One future direction is to complete a finer grained examination of goals and objectives that includes the use of non-mutually exclusive coding system (i.e., language/AAC, literacy/language) with greater differentiation within categories (i.e., expressive or receptive language, competency areas within AAC) to capture the ways that SLPs craft goals and objectives to target different skills areas simultaneously.

\section{Clinical Implications}

There are several key findings from this study that have the potential to impact clinical practice for individuals with $\mathrm{CP}$ in the schools. First, results offer support for the characterization of functional communication, as indexed by the CFCS, in addition to the usual clinical measures of impairment that are routinely used in practice. In particular, the finding that many different impairment profiles can be seen among those in the same CFCS level suggests that underlying impairments do not necessarily translate directly to functional abilities. Thus, our findings suggest that neither measures of impairment, nor measures of functional ability can or should stand alone when planning treatment for a student. Instead, both measures are necessary to provide a comprehensive picture of an individual's abilities for planning individualized interventions for students in school settings.

Our findings also indicate that across CFCS levels, school intervention is highly focused on literacy, even for the most involved students, given that literacy goals were present across all CFCS levels. This finding demonstrates an academically motivated trend in the development of school-based speech and language goals and further highlights the notion that SLPs are uniquely qualified to support teachers in the modification of literacy interventions for children with complex communication needs. School interventions were also very responsive to EF issues. However, SLPs in the school setting may be less reactive with speech and AAC needs, reserving AAC interventions for those with the most severe speech and functional communication challenges. Continuing education for SLPs regarding interventions that incorporate both speech and AAC systems and strategies may be beneficial to ensure that students with CP have the opportunity to advance their functional communication abilities to the greatest extent possible in school. 


\section{Acknowledgements}

The authors thank the children and families who participated in this research as well as the graduate and undergraduate students in the Wisconsin Intelligibility, Speech, and Communication Lab of the University of Wisconsin-Madison who assisted in data collection and data reduction. This study was funded by grant R01DC009411 from the National Institute on Deafness and Other Communication Disorders, National Institutes of Health. Support was also provided by a core grant to the Waisman Center, U54 HD090256, from the National Institute of Child Health and Human Development, National Institutes of Health. The content is solely the responsibility of the authors and does not necessarily represent the official views of the $\mathrm{NIH}$. The authors have no financial relationships relevant to this article to disclose. The authors have no conflicts of interest to disclose.

\section{References}

Ali, A., Rasheed, A., Siddiqui, A. A., Naseer, M., Wasim, S., \& Akhtar, W. (2015). Non-parametric test for ordered medians: The Jonckheere Terpstra test. International Journal of Statistics in Medical Research, 4(2), 203-207.

ASHA. (2016). Scope of practice in speech-language pathology.

Bax, M., Tydeman, C., \& Flodmark, O. (2006). Clinical and MRI correlates of cerebral palsy: The European cerebral palsy study. Journal of American Medical Association, 296(13), 1602-1608.

Bishop, D. V. (2003). Test for Reception of Grammar (TROG-2). Pearson Assessment.

Bourke-Taylor, H., Cotter, C., Johnson, L., \& Lalor, A. (2018). Belonging, school support and communication: Essential aspects of school success for students with cerebral palsy in mainstream schools. Teaching and Teacher Education, 70, 153-164.

Carrow-Woolfolk, E. (1999). Test for Auditory Comprehension of Language (3rd Ed.). Pro-Ed.

Choi, J. Y., Park, J., Choi, Y. S., Goh, Y.-r., \& Park, E. S. (2018). Functional communication profiles in children with cerebral palsy in relation to gross motor function and manual and intellectual ability. Yonsei medical journal, 59(5), 677-685.

Coleman, A., Weir, K., Ware, R. S., \& Boyd, R. (2015). Predicting functional communication ability in children with cerebral palsy at school entry. Developmental Medicine \& Child Neurology, 57(3), 279-285.

Compagnone, E., Maniglio, J., Camposeo, S., Vespino, T., Losito, L., De Rinaldis, M., Gennaro, L., \& Trabacca, A. (2014). Functional classifications for cerebral palsy: correlations between the gross motor function classification system (GMFCS), the manual ability classification system (MACS) and the communication function classification system (CFCS). Research in developmental disabilities, 35(11), 2651-2657.

Daunhauer, L. A., Gerlach-McDonald, B., Will, E., \& Fidler, D. J. (2017). Performance and ratings based measures of executive function in school-aged children with Down syndrome. Developmental neuropsychology, 42(6), 351-368. 
Dunn, L. M., \& Dunn, D. M. (2007). Peabody Picture Vocabulary Test, Fourth Edition. Psychological Corporation.

Eliasson, A. C., Krumlinde-Sundholm, L., Rosblad, B., Beckung, E., Arner, M., Ohrvall, A. M., \& Rosenbaum, P. L. (2006). The Manual Ability Classification System (MACS) for children with cerebral palsy: scale development and evidence of validity and reliability. Developmental Medicine \& Child Neurology, 48, 549-554.

Farquharson, K., Tambyraja, S. R., Justice, L. M., \& Redle, E. E. (2014). IEP goals for school-age children with speech sound disorders. Journal of Communication Disorders, 52, 184-195.

Geytenbeek, J. J., Mokkink, L. B., Knol, D. L., Vermeulen, R. J., \& Oostrom, K. J. (2014, Sep). Reliability and validity of the C-BiLLT: a new instrument to assess comprehension of spoken language in young children with cerebral palsy and complex communication needs. Augment Altern Commun, 30(3), 252-266. https://doi.org/10.3109/07434618.2014.924992

Gioia, G. A., Isquith, P. K., Guy, S. C., \& Kenworthy, L. (2000). Behavior rating inventory of executive function. Psychological Assessment Resources.

Gioia, G. A., Isquith, P. K., Guy, S. C., \& Kenworthy, L. (2015). Behavior rating inventory of executive function, second edition. Psychological Assessment Resources.

Hidecker, M. J., Paneth, N., Rosenbaum, P. L., Kent, R. D., Lillie, J., Eulenberg, J. B., Chester, K., Jr., Johnson, B., Michalsen, L., Evatt, M., \& Taylor, K. (2011). Developing and validating the Communication Function Classification System for individuals with cerebral palsy. Dev Med Child Neurol, 53(8), 704-710. https://doi.org/10.1111/j.1469-8749.2011.03996.x

Hidecker, M. J. C., Slaughter, J., Abeysekara, P., Ho, N. T., Dodge, N., Hurvitz, E. A., Workinger, M. S., Kent, R. D., Rosenbaum, P., \& Lenski, M. (2018). Early predictors and correlates of communication function in children with cerebral palsy. Journal of child neurology, 33(4), 275285.

Himmelmann, K., Lindh, K., \& Hidecker, M. J. (2013, Nov). Communication ability in cerebral palsy: a study from the CP register of western Sweden. Eur J Paediatr Neurol, 17(6), 568-574. https://doi.org/10.1016/j.ejpn.2013.04.005

Hochberg, Y. (1988). A sharper Bonferroni procedure for multiple tests of significance. Biometrika, 75(4), 800-802.

Hustad, K. C., \& Miles, L. K. (2010). Alignment between augmentative and alternative communication needs and school-based speech-language services provided to young children with cerebral palsy Early Childhood Services, 3, 129-140.

Hustad, K. C., Oakes, A., McFadd, E., \& Allison, K. M. (2016a). Alignment of classification paradigms for communication abilities in children with cerebral palsy. Developmental Medicine \& Child Neurology, 58(6), 597-604. https://doi.org/10.1111/dmcn.12944 
Hustad, K. C., Oakes, A., McFadd, E., \& Allison, K. M. (2016b). Alignment of classification paradigms for communication abilities in children with cerebral palsy. Dev Med Child Neurol, 58(6), 597604. https://doi.org/10.1111/dmcn.12944

Klang, N., Rowland, C., Fried-Oken, M., Steiner, S., Granlund, M., \& Adolfsson, M. (2016). The content of goals in individual educational programs for students with complex communication needs. Augmentative and Alternative Communication, 32(1), 41-48.

Kristoffersson, E., Dahlgren Sandberg, A., \& Holck, P. (2020). Communication ability and communication methods in children with cerebral palsy. Developmental Medicine \& Child Neurology.

Majnemer, A., Shevell, M., Law, M., Birnbaum, R., Chilingaryan, G., Rosenbaum, P., \& Poulin, C. (2008). Participation and enjoyment of leisure activities in school-aged children with cerebral palsy. Developmental Medicine \& Child Neurology, 50(10), 751-758.

Mangeot, S., Armstrong, K., Colvin, A. N., Yeates, K. O., \& Taylor, H. G. (2002). Long-term executive function deficits in children with traumatic brain injuries: Assessment using the Behavior Rating Inventory of Executive Function (BRIEF). Child Neuropsychology, 8(4), 271-284.

McCormack, J., McLeod, S., Harrison, L. J., \& McAllister, L. (2010). The impact of speech impairment in early childhood: Investigating parents' and speech-language pathologists' perspectives using the ICF-CY. Journal of Communication Disorders, 43(5), 378-396.

McCormack, J., McLeod, S., McAllister, L., \& Harrison, L. J. (2009). A systematic review of the association between childhood speech impairment and participation across the lifespan. International Journal of Speech-Language Pathology, 11(2), 155-170.

McHugh, M. L. (2012). Interrater reliability: the kappa statistic. Biochemia medica: Biochemia medica, 22(3), 276-282.

Mehraban, A. H., Hasani, M., \& Amini, M. (2016). The comparison of participation in school-aged Cerebral Palsy children and normal peers: A preliminary study. Iranian journal of pediatrics, 26(3).

Mei, C., Reilly, S., Bickerton, M., Mensah, F., Turner, S., Kumaranayagam, D., Pennington, L., Reddihough, D., \& Morgan, A. T. (2020). Speech in children with cerebral palsy. Developmental Medicine \& Child Neurology, 62(12), 1374-1382.

Mei, C., Reilly, S., Reddihough, D., Mensah, F., \& Morgan, A. (2014, Aug). Motor speech impairment, activity, and participation in children with cerebral palsy. Int J Speech Lang Pathol, 16(4), 427435. https://doi.org/10.3109/17549507.2014.917439

Nathan, L. (2002). Functional communication skills of children with speech difficulties: Performance on Bishop's Children's Communication Checklist. Child Language Teaching and Therapy, 18(3), 213-231.

Palisano, R., Rosenbaum, P. L., Walter, S., Russell, D., Wood, E., \& Galuppi, B. (1997). Development of the gross motor function classification system. Developmental Medicine \& Child Neurology, $39,214-223$. 
Palisano, R. J., Avery, L., Gorter, J. W., Galuppi, B., \& McCoy, S. W. (2018). Stability of the gross motor function classification system, manual ability classification system, and communication function classification system. Developmental Medicine \& Child Neurology, 60(10), 1026-1032.

Pennington, L., Dave, M., Rudd, J., Hidecker, M. J. C., Caynes, K., \& Pearce, M. S. (2020). Communication disorders in young children with cerebral palsy. Developmental Medicine \& Child Neurology, 62(10), 1161-1169.

Pennington, L., Virella, D., Mjoen, T., da Graca Andrada, M., Murray, J., Colver, A., Himmelmann, K., Rackauskaite, G., Greitane, A., Prasauskiene, A., Andersen, G., \& de la Cruz, J. (2013). Development of The Viking Speech Scale to classify the speech of children with cerebral palsy. Research in Developmental Disabilities, 34(10), 3202-3210. https://doi.org/10.1016/j.ridd.2013.06.035

Raghavendra, P., Virgo, R., Olsson, C., Connell, T., \& Lane, A. E. (2011). Activity participation of children with complex communication needs, physical disabilities and typically-developing peers. Developmental Neurorehabilitation, 14(3), 145-155.

Ranganathan, P., Pramesh, C., \& Aggarwal, R. (2017). Common pitfalls in statistical analysis: Measures of agreement. Perspectives in clinical research, 8(4), 187.

Roid, G., \& Miller, L. (1997). Leiter International Performance Scale-Revised. Stoelting Company.

Sakash, A., Broman, A. T., Rathouz, P. J., \& Hustad, K. C. (2018). Executive function in school-aged children with cerebral palsy: Relationship with speech and language. Research in Developmental Disabilities, 78, 136-144. https://doi.org/10.1016/i.ridd.2018.05.015

Soriano, J. U., \& Hustad, K. C. (2021). Speech-language profile groups in school aged children with cerebral palsy: Nonverbal cognition, receptive language, speech intelligibility, and motor function. Developmental Neurorehabilitation, 24(2), 118-129.

Virella, D., Pennington, L., Andersen, G. L., Andrada, M. d. G., Greitane, A., Himmelmann, K., Prasauskiene, A., Rackauskaite, G., De La Cruz, J., \& Colver, A. (2016). Classification systems of communication for use in epidemiological surveillance of children with cerebral palsy. Developmental Medicine \& Child Neurology, 58(3), 285-291.

Westby, C., \& Washington, K. N. (2017). Using the International Classification of Functioning, Disability and Health in assessment and intervention of school-aged children with language impairments. Language, Speech, and Hearing Services in Schools, 48(3), 137-152.

World Health Organization. (2007). International Classification of Functioning, Disability, and Health: Children \& Youth Version: ICF-CY. World Health Organization. 